\title{
SYNTHESIS OF MANUFACTURING AND FACILITY DATA FOR SUSTAINABILITY ANALYSIS
}

\author{
John Michaloski, Goudong Shao, \\ Frank Riddick, and Swee Leong \\ National Institute of Standards \\ and Technology \\ Gaithersburg, Maryland, USA
}

\author{
Jonatan Berglund \\ Chalmers University of Technology \\ Gothenburg, Sweden
}

\author{
Jorge Arinez and Stephan Biller \\ General Motors \\ Warren, Michigan, USA
}

\begin{abstract}
This paper discusses data synthesis of production and facility knowledge for sustainability analysis by applying the ISA95 "Activity Models of Manufacturing Operations Management" (MOM) model. Presently, production and facility management basically function independently of each other. This paper presents the addition of facility activities to the MOM model, in accordance with the needs for attaining a holistic view of sustainability analysis. Historically, production and facility data are represented in various forms, e.g., data bases, CAD, and spreadsheets, without a common unifying representation. Based on this combination of incompatible modeling tools, the use of Core Manufacturing Simulation Data (CMSD) is proposed as a standard framework for integrating the broad range of technology. A case study of the data synthesis for a precision sand casting production facility is explored.
\end{abstract}

\section{Keywords}

Sustainability analysis, precision sand casting, finite state machine, sustainable manufacturing, data synthesis, energy efficiency, Key Performance Indicator

\section{Nomenclature}

CAD Computer Aided Design

CMSD Core Manufacturing Simulation Data Standard

CNC Computer Numerical Control

DES Discrete Event Simulation
EMS Energy Management System

ERP Enterprise Resource Planning

FSM Finite State Machine

IAQ Indoor Air Quality

HVAC Heating, Ventilating, and Air Conditioning

KPI Key Performance Indicators

MES Management Execution System

MESA Manufacturing Enterprise Solutions Association

MOM Manufacturing Operations Management

MTBF Mean Time between Failures

MTTR Mean Time to Repair

OEE Overall Equipment Effectiveness

PLC Programmable Logic Controller

ROI Return on Investment

SPC Statistical Process Control

XML eXtensible Markup Language

\section{Introduction}

Better integration of process and energy data offers a significant sustainable opportunity to reduce manufacturing energy consumption $[1,2]$. The lower cost of networks and computers has enabled plants to implement information systems that can perform real-time data collection and archiving of the operational behavior of their facility and plant operation. Increasingly, companies collect process and energy data from the various control and supervisory systems on the plant floor.

Although process and energy data collection is routinely 


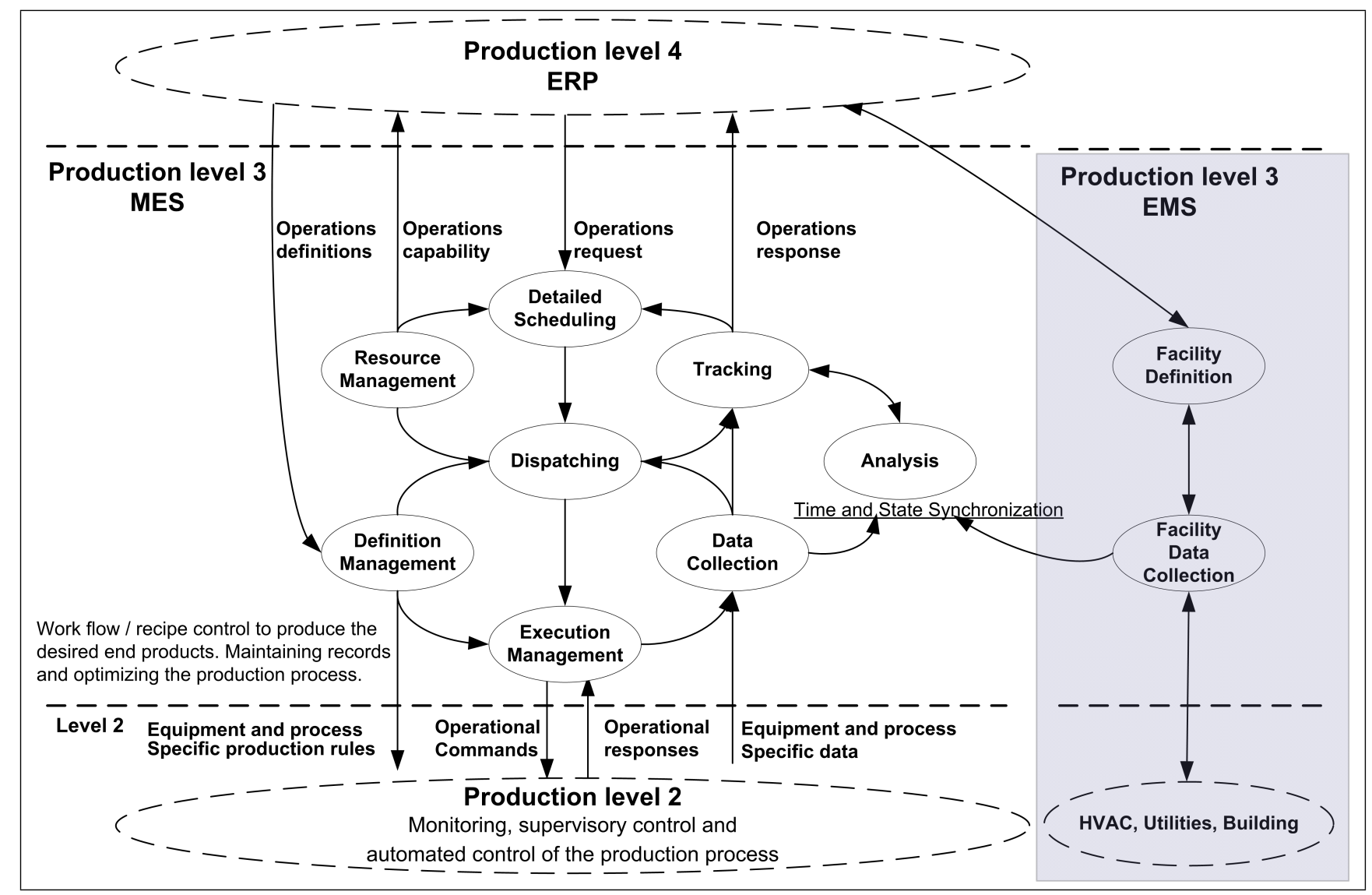

FIGURE 1. Role of Facility Management within ISA 95 Activity Framework

done, there are often many (and unconnected) data collection subsystems involved [3]. Given such disparate systems and databases, an integrated approach to synthesizing production system and process energy and facility energy is a prerequisite to offering a holistic analysis of plant operation. Production data is collected in real-time to help monitor, control, and report on manufacturing activity. In a sustainable world, process monitoring must also encompass the amount of energy required to run a machine. Facility energy consumption includes those for lighting, compressed air, and air quality control. For example, analysis within this realm would correlate and balance the requirement of HVAC against production energy, heat, and emissions. Thus, for such case, production knowledge includes design information such as machine size and location on the plant floor, its spatial relationship to HVAC air supply, as well as its dynamic heat emissions during production and temperature requirements of the part and process for satisfying part feature accuracy requirements.

Synthesizing production data must consider all aspects of the manufacturing operation, i.e., plant, process and personnel as well as design, production, and maintenance. Typically, the primary focus of improvement has been on production, through modeling and analysis of manufacturing operation, while the day-to-day facility-related operation, although important, takes on a less prominent role. Part of the problem can be attributed to the functionality separation between manufacturing execution systems (MES), which handles production activities, and facility management, specifically energy management system (EMS), which handles energy-related activities within building services. In effect, MES and EMS are "silo" operations, that is, isolated subsystems from each other.

ISA 95 Parts 1, 2, and 3 [4-6] specifications provide a standard terminology and integration framework to define system requirements between different production levels. ISA-95 Part 3 contains the "Activity Models of Manufacturing Operations Management" (MOM) model, as shown in Figure 1, which serves as the activity framework for production, inventory, maintenance, and quality within a manufacturing enterprise. The MOM model describes Level 3 in the ISA 95 multi-level functional hierarchy of enterprise-control system integration of production activities:

Production Level 4 - logistics and planning functions.

Production Level 3 - manufacturing operations management 
of shop floor work flow.

Production Level 2 - monitoring, supervisory control and automated control of the production process.

Production Level 1 - sensing and controlling the production process (not shown).

Production Level 0 - actual production process (not shown).

In the shaded box of Figure 1, we add a facility concept to the MOM model, to represent the role of facilities in developing a more holistic view of production. The role of EMS in regards to facilities and facility energy data collection is explicitly shown as new elements in the MOM model. Implicit in the MOM model is the role of process energy. The MOM model does not explicitly cover sustainability or energy efficiency, but these can be inferred activities within the Execution and Data collection activities.

Production data becomes production knowledge when it is organized, structured, and analyzed in an accessible manner such as, a SQL query on a database or data in spreadsheets, with the data meaningfully interrelated. The synthesis of data from different sources is a necessary step before reasoning and analysis. Clearly, a common information architecture that supports the sharing and synthesis of rich domain "real, digital, and virtual" knowledge is imperative for production analysis to be cost-effective [7]. In order to synthesize data into production knowledge, a neutral format to represent this data is desirable. From our perspective, the Core Manufacturing Simulation Data (CMSD) standard [8] offers the best alternative to allow the translation from numerous related domains into a manufacturing domain-specific representation suitable for sustainability analysis.

This paper studies the data synthesis requirements for better integration of production and facility knowledge for sustainable analysis. Section 2 discusses the issues related to transforming production data into production knowledge and the state machines applied within the data acquisition process. Section 3 studies sustainable manufacturing requirements analysis. In Section 4, a case study of a precision sand casting production is discussed, it is a DES model of a casting and molten delivery line, which is used to clarify the data synthesis requirement. The paper concludes with a short summary.

\section{Data Acquisition}

Informative, accurate, and timely shop-floor data should be considered vital to understanding production. Only with accurate data can analysis and benchmarking be suitably done to eliminate waste and inefficiencies. One of the most important problems when developing production knowledge is finding and synthesizing the required data. We address the problem of acquiring the necessary data to model the sustainability of manufacturing and facility systems.

Data synthesis involves the activities for obtaining accurate system input and output data and transforming the data into a unified representation that is meaningful and cost-effective for use in analysis tools. The Manufacturing Enterprise Solutions Association (MESA) points out that automated collection and use of accurate data is critical to conducting effective plant operations and processes [9]. Preferably, the data must be in the form of production event data, such as data for per state per part, not as the cumulative time equipment spends in each state, that is, total time spent in the busy versus idle state during the course of a shift.

Actual production data must be organized and filtered to increase its effectiveness. Raw data is typically filtered into event data so as to satisfy subsequent analysis requirement that will be applied, i.e., data contains events and time duration within the event. For example, Figure 2 shows a state mapping for machine utilization, with the raw data collected first as polled state queries then aggregated by time into event data. With cost per time functions assigned to each state, process and energy performance can be computed by state durations to summarize factory operation.

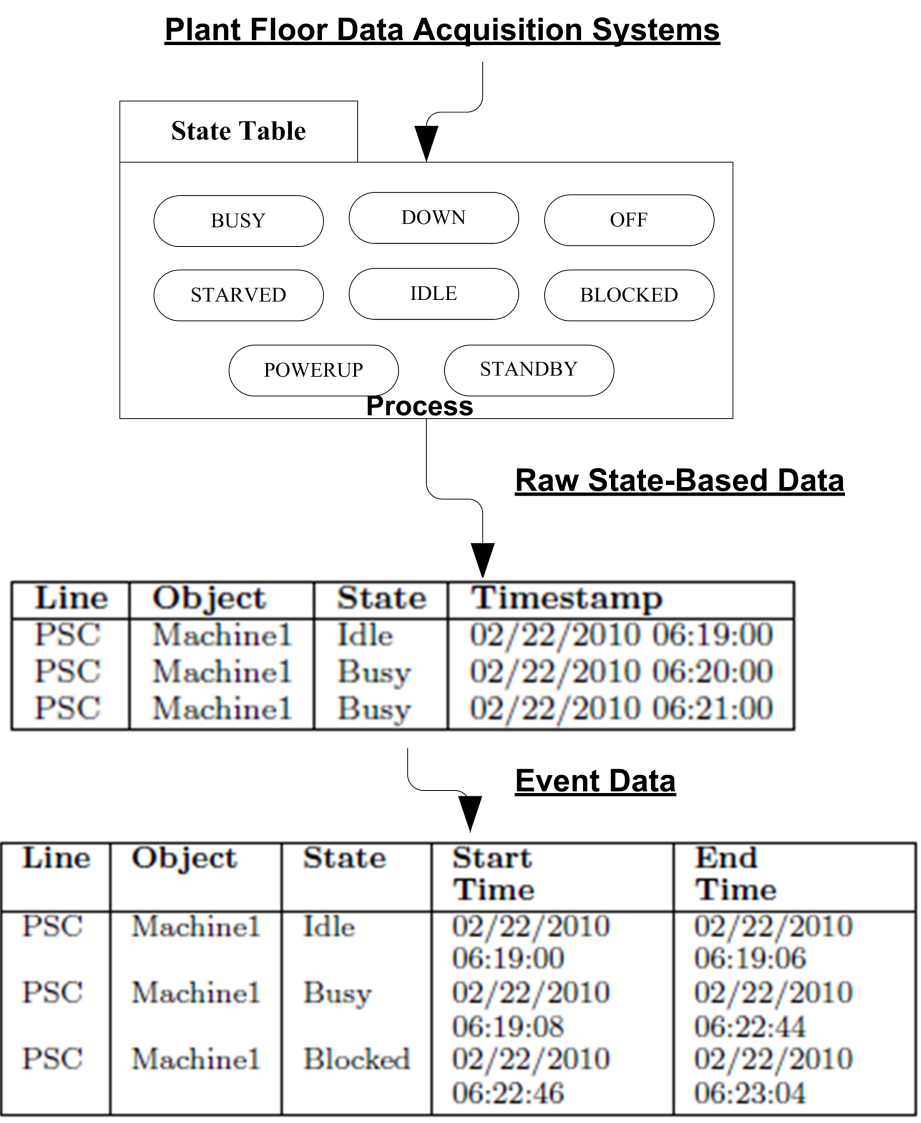

FIGURE 2. Real-time behavior modeled as Finite State Model 
Plant behavior can be modeled quite effectively using a finite state model [10]. Equipment such as fans, machinery, or lighting can be modeled by finite state machines. Figure 2 shows an example of the state model for machinery resources, where the equipment has states for OFF, BUSY, FAULT, IDLE, DOWN, STARVED, BLOCKED, POWERUP and STANDBY states. Such a model is particularly useful because it is equally applicable to both equipment used for production and by the facility. The only difference is that for facility equipment such as a light, OFF, BUSY and FAULT, are the primary states.

STANDBY involves powering the equipment into a sleep mode that saves energy by using minimal power. STANDBY is an equipment state that would be greatly beneficial to improve energy efficiency but is currently rarely used. Several industrial network groups are implementing energy awareness into their specifications $[11,12]$. Several strong use-case scenarios for networkenabled energy management based on the STANDBY state exist, which includes optimized production leading to energy savings during short, long, and unplanned pauses.

EMS does not collect energy data the same way that production data is collected. Process and facility energy data measured on its own is based on summed measurements over time to determine power consumption, but may include peaks, spikes, and other cost-sensitive parameters. For data synthesis, the key energy parameter is not only power consumed, but also the correlation between process state and the energy needs, which is not simply time-based readings, e.g., the amount of energy being consumed while production is processing, versus when the equipment is down.

\section{Sustainability Analysis}

Sustainability analysis must map objectives, constraints, data, and specifications into the requirements for the data synthesis. The objectives are to better understand these relationships to improve energy efficiency, process efficiency, part quality, yield rate, and other established production objectives.

Optimization objectives need to be carefully formulated. For example, if the objective is to reduce energy consumption, often, improving process performance corresponds to energy savings. If the production line is frequently down, the production equipment uses less power due to the idling, but over time will result in a more power required per unit yield. So, some production improvements, such as higher yield, may end up using more shortterm energy, but this is a positive thing. This implies that all aspects of potential objective functions must be studied to accurately understand performance benchmarking.

In the MOM model, Production Level 4 (the ERP) primarily gathers plant data for analysis of the accounting and materials control system. Production Level 3 may acquire the same data, but also allows analysis against plant-level performance measures. Production Level 3 aims at analyzing and improv- ing the individual process or line under control, while the same data when used for Production Level 4, aims more to analyze how effectively a given process is contributing to overall plant performance.

Production can be analyzed as a KPI by fitting event data to a statistical distribution. KPIs are metrics that provide a means to analyze productivity. KPIs can be computed for benchmarks, comparisons, estimations, and forecasts, which are derived from data pertaining to the process, the machinery and equipment, the product manufactured and its quality, the manufacturing personnel and other related manufacturing resources.

International Organization for Standardization (ISO) has developed a draft standard ISO 22400, "Automation systems and integration - Key performance indicators for manufacturing operations management," that offers guidance with the definition, usage, application and benefits of production KPI and associated metrics [13]. ISO 22400 offers metrics in understanding the performance of MES functionality at Level 3 of the MOM model, along with collective subordinate operations, including detailed scheduling, resources management, procedure management, data collection, tracking, analysis and reporting. Of relevance to sustainability, ISO identifies five KPIs - emission ratio, energy ratio, ratio of used material, harmful substances, and hazardous waste.

Measured KPI are necessary, but not sufficient, in order to enact improvements. To be sufficient, the KPI also require a detailed analytical breakdown of the measurement to understand causality of the measurement. For example, throughput is a KPI to measure the performance of a process, i.e., the quantity per unit time is produced and is an important indicator of production efficiency. Although, throughput KPI forms a necessary basis for improvements by better production information, unsatisfactory KPI values implies the need for more detailed data analysis that is not directly discernible from the KPI itself. Instead, production data of finer granularity is required to understand the contributing factors that lead to the unsatisfactory KPI. For example, in one scenario a machine often sits idle blocked, waiting for the arrival of materials or tooling. In contrast, excessive machine or process faults cause prolonged unproductive periods. In either case, understanding the type and severity of delays or faults within production is required to remediate process problems and improve OEE. Often the underlying analysis is more difficult to undertake and the data is more difficult to categorize and remediate.

To understand the complicated manufacturing interrelationships, Figure 3 shows a systematic approach that allows for the data synthesis and analysis using multiple software tools to understand and improve production. A review of the MOM Level 3 and 4 function data requirements and the data, KPI performance metrics within these elements follows.

ERP goal is to maximize ROI and quality, and does this through monitoring and analyzing job, product and inventory costs. 


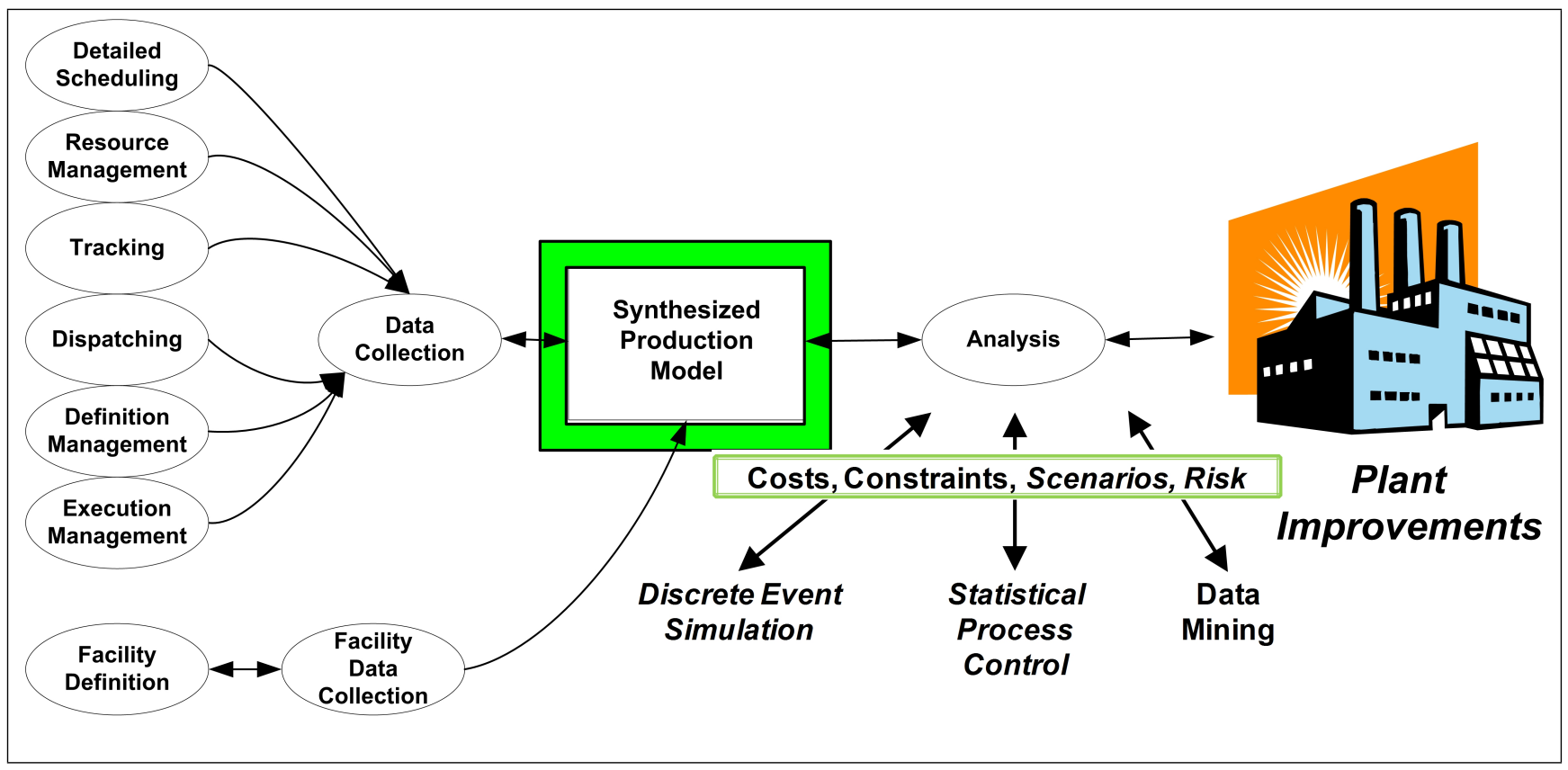

FIGURE 3. Synthesis of Production System, Process Energy, and Facility Energy Data for Continuous Improvement

Facility EMS goal is to supply water, power, compressed air, and maintain a healthy IAQ. Data is collected to analyze energy consumption, energy losses, and indoor environment (temperature, humidity, emissions, heat generation, and dust).

Resources cover machines, tools, labor, specific abilities, materials, and supporting equipment, with the goal of high OEE and reliable operation. Dynamic data includes cycle time, MTBR, MTTR, and process energy consumption. Design data includes size, location, model, buffer sizes, costs, and utility data as well as equipment performance attributes, e.g., machine tool rated horsepower, autonomous vehicle or conveyor speeds.

Definition management handles part programs or recipes and collects and analyzes production data for cycle times and for feedback into product design development.

Execution manages and controls production automation. It collects and analyzes process data for optimizing throughput, yield, machine and process efficiencies, waste (scrap cost), maintenance, scheduling, part quality.

Tracking collects and analyzes quality assurance data based on deviations from the ideal part characterization.

For larger scale production, the integration of sustainability knowledge from a variety of sources is a challenge. Even a medium sized production line could contain thousands of devices and tools, both discrete and continuous operations, building and layout parameters, as well as HVAC equipment. Assuming enough sustainable knowledge is available, a neutral format to represent this data is desirable. Synthesizing manufacturing knowledge is difficult since all the necessary bits and pieces of production data can be in different file formats and representations. Digital CAD files are used as "drawings" for the facilities and plant layout, process plans are contained in data bases, and workflow data in spreadsheets. So, various pieces of production knowledge may be distributed throughout the enterprise. Often, storage of the production knowledge is tailored for human comprehension, (i.e., spreadsheets), that are not as conducive for interoperable software sharing.

Because of this breadth and disparity of information and sources, a move towards a common repository format with one unifying and neutral information model would be of great benefit to facilitate sustainability modeling and analysis. CMSD is a standard specification that would allow the translation from numerous related domains into a manufacturing domain-specific representation suitable for sustainability analysis [8]. The goal of CMSD is to facilitate the exchange of information between simulation models and other manufacturing software applications, such as used in process planning, scheduling, inventory management, production management, or supply chain management. The CMSD specification focuses on core modeling concepts shared by all manufacturers and provides a generic property structure to adapt to various specialized functional requirements. The CMSD information model covers the major manufacturing aspects including Organization, Resource, Skill, Setup, Operation, Maintenance, Part Models, Bill-of-materials, Inventory, Process Plans, Schedules, and Statistical modeling aspects. 


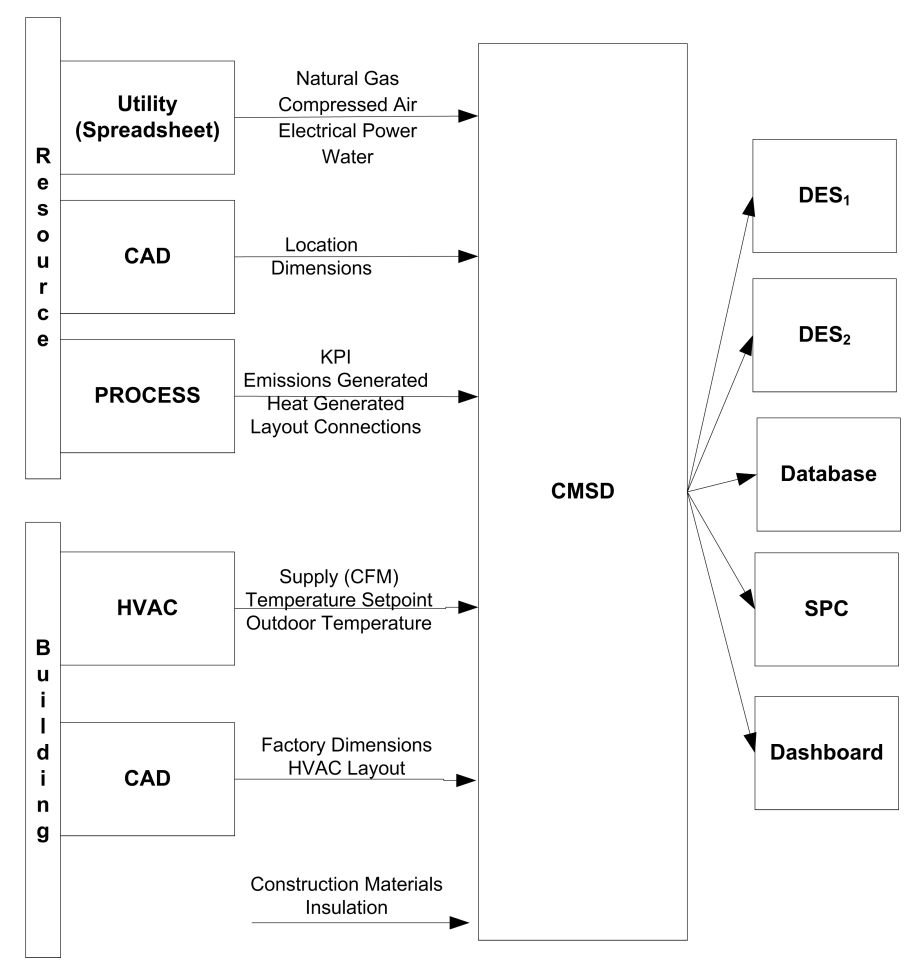

FIGURE 4. Knowledge Fusion into CMSD Representation

Based on XML, CMSD provides a standard format that contains the basic data required to represent the sustainable information while simultaneously allowing additional, detailed, application-specific data to be contained without invalidating the format. XML has gained acceptance as a common export format from internal proprietary formats. Converting production knowledge and process data into a common repository based on a CMSD neutral representation is valuable, and has been applied previously to manufacturing analyses [14-16]. The use of automated technology to extract production data from various data sources and transforming the data into a CMSD will help to reduce the time and cost to implement factory floor analysis [17]. Figure 4 shows the strategy of using CMSD to convert production knowledge and process data into a common repository with a neutral representation so that it can be easily transformed into other tools, for example, DES, database, or SPC.

Effective manufacturing analysis tools include SPC, DES and Data Mining. A DES models a system as a chronological sequence of discrete events and is useful for modeling and analysis of manufacturing system issues, such as, machinery operation, shop workflow and scheduling, and production lines. A DES can model and evaluate real or proposed production concepts, identify problem areas, quantify and optimize system performance. Simulation is especially popular where the complexity of the real world makes analytical closed-form solutions difficult. Data mining combines statistics and artificial intelligence to find rules that are contained within data $[18,19]$. Data mining methods include classification, estimation, prediction, clustering, and affinity grouping. SPC uses statistical methods to measure and evaluate production variability in order to improve the production process and ensure quality, minimize process costs due to scrap and rejects, and minimize the cost of customer dissatisfaction with poor products.

Production analysis can be used to uncover opportunities for optimizations, savings and costs, as well as mitigate risks, and help avoid potential crisis points. For example, analysis scenarios could be developed to understand the implications of energy usage during production stoppages, to understand the effect of changing production schedules, or to see what can be done to lower the risk associated with rising energy costs or energy shortages.

\section{Case Study}

A case study that investigates the data requirements for production/facility analysis was performed for a precision sand casting facility that produces aluminum engine blocks. Sand casting can produce parts ranging from a few ounces to several tons, and is relatively inexpensive, and acceptably precise, with dimensional control in the range of $\pm 0.8 \mathrm{~mm}$ to $3.2 \mathrm{~mm}$ ) [20]. Sand casting is one of the most energy-intensive industries, and is a prime candidate for energy analysis. The primary elements in the sand casting facility are described below:

Melting Aluminum - involves transforming incoming solid aluminum in various forms (virgin material, machining chips, gates, sprues, etc.) into the molten state and maintaining it at the required temperature until it is ready to be poured. During melting and holding, the molten aluminum is refined and the chemistry adjusted as necessary to achieve proper alloy quality characteristics. Once molten, the aluminum is degassed, leveled, and laundered to remove deleterious gases before being tapped to flow into cores.

Core Making - produces the sand cores. Core parts are molded from sand and binding elements, assembled into the engine block core, and then dried before casting. Since sand casting is an expendable mold metal casting process, the core process builds a new sand core for each casting.

Casting and Finishing - consists of pouring the molten aluminum into the sand cast core, after which, the casting is cooled and the casting sand is removed from the solidified aluminum engine block by shakeout, trim, and degating operations.

For confidentiality, the actual performance data has been normalized, however, it is representative of the data that is often frequently encountered in facilities such as the one described in this study. The precision sand casting operation involves hundreds of pieces of electrical equipment, which include 


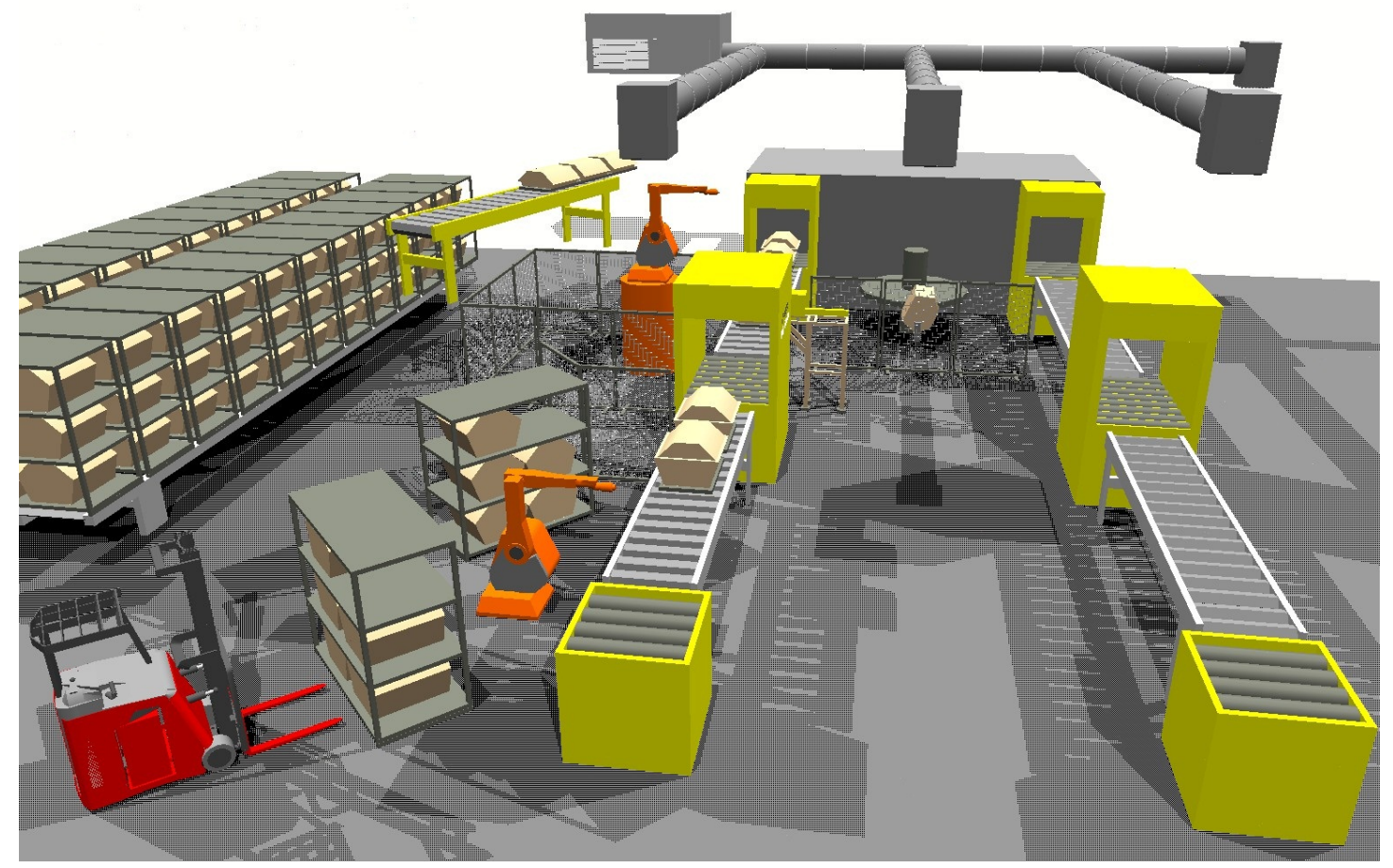

FIGURE 5. Simulation Model of Casting Operation.

robots, conveyors, elevators, sand core making machines, and saws. The precision casting operation under study uses 23,225 square meters (250,000 square feet) of a larger facility to make polyurethane cold box sand cores that are assembled into 700 pound molds. There are three mold, casting, and finishing lines, with over one thousand pieces of equipment as part of the manufacturing process. The extent of the casting production size necessitated narrowing the initial analysis scope to one of the molten aluminum delivery and cast filling lines. The analysis was limited to data already being collected by the plant's production system.

To understand the complicated relationships between the data synthesis and the analysis requirements, we used DES modeling of production and facility energy as it is well-suited to handle the complexity of large scale interaction of building, machines, and processes. The facility utility and the performance data were mapped into CMSD, and using various open-source and DES software the data was synthesized. Mapping of the facility layout into CMSD using the CAD representation was considered but without enough semantic content to understand, was considered too time-consuming and a more manual approach was used.

The scope of the study covers the molten aluminum delivery system and the pouring of aluminum into engine casts. The pouring process also covers the immediate handling of the casts prior and after the point of the pouring. Figure 5 shows a DES screen capture of the model, which includes one casting and molten aluminum line and its relationship to the facility HVAC system. The model includes the processes of preparation of the preassembled casts, i.e., addition of alloys and chill inserts prior to pouring and extraction of chill inserts after the pouring.

In further detail, the casting process starts with an elevator transferring in recycled fixture plates to undergo another round of prepare-fill-cool. A fixed number of fixtures cycle through prepare-fill-cool phase cycles in production, such that, for the casting operation, a recycled fixture is placed on conveyor. Next a robot places a core package onto a fixture plate to be transferred along the conveyor for each stage of the casting operations. The delivery of core packages is sequenced from an automated storage rack, with each rack having room for up to six core packages. The storage rack is replaced with a new storage rack when all six have been picked and placed onto a fixture plate. The first operation on the casting transfer line does a chill insert into the cavity wall. Chills are embedded in the mold cavity wall to control the solidification process [20]. Then the core package moves along conveyor where some final alloys are inserted into the mold and a robot places a cover top on the core package. A gantry crane lifts the core package into a receiving area where the core is filled with molten aluminum. After being filled, the fixture and core package is lifted and placed onto conveyor by another gantry crane to begin the cooling process. The first stop is to remove the chill plate from the core. Then the cast moves onto the chill- 
ing transfer line where the sand core is removed from the cast via shakeout operations, and later the cast is trimmed and degated.

In the plant under study, production system and process energy data are routinely archived into databases. Data handling operations, such as filtering of the raw data into event data and cleansing of the data, are required, as in any modeling work. Production data can be described by raw, cumulative event-based, or statistical distribution parameters. The granularity of the actual precision casting data was limited to process steps in a work cell (and not by an individual piece of equipment), which includes a group of related equipment.

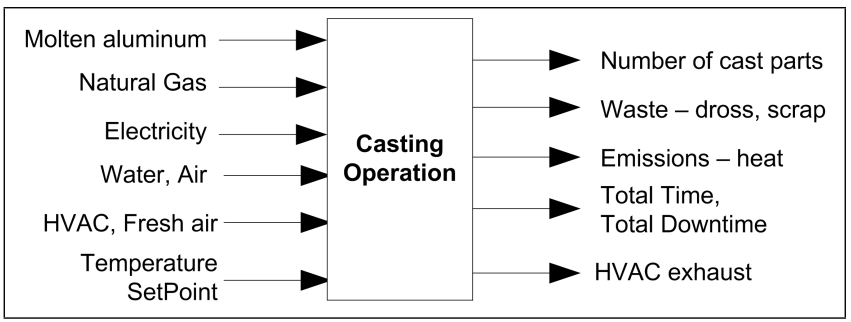

FIGURE 6. Measurable Input/Output of Casting Operation

The first step in data synthesis for the casting operations was to collect relevant high-level data. Some of the data was easily accessible. Other data was unavailable or available but uncorrelated to production. Figures 6 shows a model that describes the measured inputs, i.e., molten aluminum, natural gas, electricity, HVAC fresh air and temperature setpoint. The measured outputs included casts produced, casts scrapped, accumulated dross, and cycle time statistics. Data such as machine and molten emissions were estimated, but the uncertainty of estimations was unclear. HVAC air supply and temperature setpoint was available, but difficult to correlate to production. Other performance analysis statistics, such as daily throughput and yield, can easily be calculated from the basic operation itemization.

Within the facility monitoring, the electrical and natural gas consumption was metered and data was collected every 15 minutes. Because there was no production overnight but the existing molten aluminum remained in the delivery system, we were able to estimate the energy necessary to maintain the aluminum in a molten state, and then use energy readings during production to estimate consumption. From this we were able to calculate the energy consumption per cast. However, because the energy data was accumulated at the factory level, it was difficult to analyze finer process-level energy consumption, such as, for understanding the effect of line sequencing delays from down equipment. Analysis of the given summary energy data finds that the molten process dominates energy consumption. Overall the energy data at this level of granularity was found to be useful for understanding some risk scenarios - such as analyzing costs if the prices of electricity or natural gas changed.

Refocusing from the plant level to cell level production, process data is continuously collected from PLCs throughout the plant. Raw cycle time for casting and equipment utilization and fault data was collected and adapted into process state-based KPI parameters. The daily production plan was based on fulfilling a daily cast quota in one shift, no matter how long it took. This leads directly to cumulative statistical data based on daily shift output per number of casts. Within the cast line, the number of casts, molten aluminum delivery, and waste in combination were useful in assessing yield and data validity.

\section{Discussion}

Data quality issues can severely hamper the effectiveness of sustainability analysis and poor data issues can be pervasive and costly [21]. Data quality has several dimensions in which to measure and assess the accuracy. The data dimensions of interest we found in our case study included:

1. Accuracy - measures the agreement between the recorded and the actual value,

2. Timeliness - measures the latency between collecting and using the data, and given some cutoff can be used to determine if the data is out of date,

3. completeness, measures the difference between the set of recorded values against the set of actual values,

4. Consistency - measures data values for correctness and completeness in all cases

5. Interpretation - provides for any expected differences from the expected scenario, and

6. Interdependency - measures the relationship between two independent pieces of data.

The specific data quality issues uncovered within the case study included:

Interpretation - the casting facility uses a shifting break schedule, so the machines are never idle for operator breaks.

Consistency - the time accounting between the various production states, that is, times for Down, Blocked, Starved, and Total time for production Processing were collected for most machines. By definition, one expects the following time relationship to be immutable:

$$
T_{\text {Total }} \geq T_{\text {Downtime }}+T_{\text {Blocked }}+T_{\text {Starved }}
$$

where $T_{S}$ is the time spent in state $S \in$ \{Downtime Blocked Starved $\}$ and $T_{\text {Total }}$ is the total measured production time. However, although impossible, some days this relationship was false. 


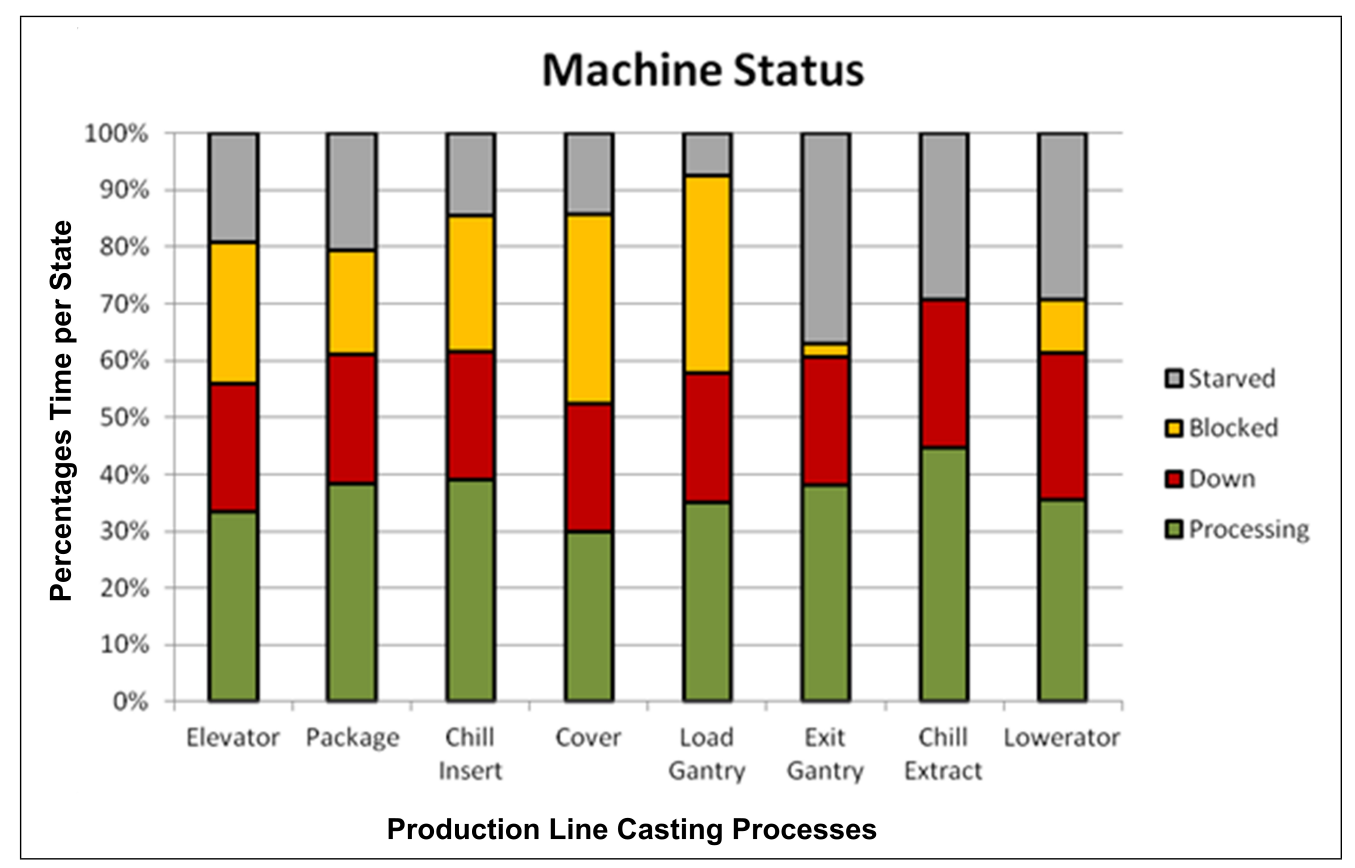

FIGURE 7. FSM Breakdown of Steps in the Casting Operation

Interdependency - although data for steps in the production line are clearly independent of each other, some of the interstep data displayed a linked interdependency. i.e., consecutive stations have identical down/repair times. This data irregularity was accounted for in the modeling.

Accuracy - fault data for downtimes was found to be skewed by production during overnight and over weekends breaks. Fault data was aggregated from the raw event plant data, however, a machine break down occurring at the end of the day, skewed the data. For example, a fault at 5:55 pm Friday (Feb 23rd) that is repaired by 07:15 am (Feb 24th, will show an aggregated data downtime of: 23rd: $6 \mathrm{hrs} 5 \mathrm{mn}$ and 24th: $7 \mathrm{hrs} 15 \mathrm{mn}$. In reality, the shift ended at 6:30 and machine was repaired immediately and could be started as normal the following day. Actual downtime: 23rd: $35 \mathrm{mn}$ and for the 24th: $0 \mathrm{mn}$.

Clearly, bad data leads to bad decisions in manufacturing. Further, data should never just be assumed to be correct. But with some effort bad data can be remediated, and analysis results verified as reliable. For example, from our study Figure 7 shows the statuses of cells/processes in the casting operations, where data is collected during scheduled production time. As we can see, the bottleneck appears to exist between the loading and unloading of the gantry, where the pouring occurs. The conclusion to be drawn from this analysis is that pouring may be a problem, and could require further monitoring. However upon further indepth analysis, it was determined that failure data for the pouring was overstated due to inconsistencies discussed previously in the raw data gathered and aggregated.

Throughout the case study analysis, there are several points of execution where such errors can be introduced and it is important to continuously verify and validate data so that the DES analysis results are indeed valid.

Bigger problems were the lack of data or the poor granularity of data, and the reliance on estimation. Synchronizing energy data to the process within the plant was difficult since energy collection is integrated over time, and energy collection was uncorrelated to process performance. This means that energy data needs to be transformed from timed into state-based power consumption. Unfortunately, the energy data was not of fine enough time granularity to make the transformation meaningful. It would be expedient if the energy and the raw process data were logged together simultaneously in a database. Managing data merges that are based on inconsistent data dimensions are prone to errors and is a time-consuming task.

\section{Summary}

Faced with ongoing competitive demands, manufacturers are in a continual struggle to optimize production and sustainable manufacturing. This paper looked at improving production with better sustainability by understanding the energy control issues within manufacturing and the facility. Better visibility and understanding of production can help to attain world-class production and energy control and management. However, evaluating the performance of a manufacturing system and processes 
requires collecting and integrating the correct and accurate process data in order to analyze performance. We introduced an augmented ISA 95 MOM model that included sustainable building aspects to better understand the complex sustainability issues of data synthesis, the relationship to analysis requirements, and the problems and verification required to achieve success. The augmented ISA $95 \mathrm{MOM}$ with sustainable building aspects serves as a helpful reference model to those trying to understand a holistic view of manufacturing sustainability, especially pertinent when you consider that manufacturing in the United States uses onethird of all the energy consumed and that buildings constitute about $39 \%$ of all energy use [22].

In the future, additional research will be done to better understand automatically matching analysis requirements with data requirements. For example, if attempting to analyze energy consumption based on equipment heat emissions, then CAD layout data, equipment power ratings, and HVAC data would be required. In the short term, better data validation techniques would be useful to insure that analysis results are accurate.

\section{Disclaimer}

Commercial equipment and software, many of which are either registered or trademarked, are identified in order to adequately specify certain procedures. In no case does such identification imply recommendation or endorsement by the National Institute of Standards and Technology or General Motors, nor does it imply that the materials or equipment identified are necessarily the best available for the purpose.

\section{REFERENCES}

[1] Arinez, J., and Biller, S., 2009. "Innovations in energy measurement and control for manufacturing systems". In NIST National Workshop on Challenges to Innovation in Advanced Manufacturing: Industry Drivers and R\&D Needs.

[2] Arinez, J., and Biller, S., 2010. "Integration requirements for manufacturing-based energy management systems". In 2010 IEEE PES Conference on Innovative Smart Grid Technologies.

[3] Arinez, J., Biller, S., Lyons, K., Leong, S., Shao, G., Lee, B. E., and Michaloski, J., 2010. "Benchmarking production system, process energy, and facility energy performance using a systems approach". In Performance Metrics for Intelligent Systems Workshop (PerMIS '10), IEEE.

[4] ISA, 2000. ISA-95.00.01-2000, Enterprise-Control System Integration Part 1: Models and Terminology. International Society of Automation.

[5] ISA, 2001. ISA-95.00.02-2001, Enterprise-Control System Integration Part 2: Object Model Attributes. International Society of Automation.
[6] ISA, 2005. ANSI/ISA-95.00.03-2005, Enterprise-Control System Integration, Part 3: Models of Manufacturing Operations Management. International Society of Automation.

[7] Lanz, M., and Tuokko, R., 2009. "Generic reference architecture for digital, virtual, and real representations of manufacturing systems". In Proceedings of the Indo-US Workshop on Designing Sustainable Products, Services, and Manufacturing Systems.

[8] Simulation Interoperability Standards Organization (SISO), 2010. Core manufacturing simulation data (CMSD) - siso-std-008-2010. Accessed December 3, 2011. http: / / www. sisostds . org.

[9] MESA Inernational, 1997. Execution-Driven Manufacturing Management for Competitive Advantage - White Paper. Manufacturing Enterprise Solutions Association.

[10] ISA, 2008. ISA-TR88.00.02-2008 Machine and Unit States: An Implementation Example of ISA-88. International Society of Automation.

[11] ODVA, 2011. Optimization of Energy Usage: ODVAs Vision of Energy Optimization for the Industrial Consumer. Ann Arbor, MI.

[12] PROFINET, 2011. Common application profile PROFIenergy technical specification for PROFINET.

[13] ISO, 2011. ISO/DIS 22400-2 Automation systems and integration - Key performance indicators for manufacturing operations management - Part 2: Definitions and descriptions. International Organization for Standardization, Geneva, Switzerland.

[14] Heilala, J., Vatanen, S., Tonteri, H., Montonen, J., Lind, S., Johansson, B., and Stahre, J., 2008. "Simulation-based sustainable manufacturing system design". In WSC '08: Proceedings of the 40th Conference on Winter Simulation, Winter Simulation Conference, pp. 1922 - 1930.

[15] Hazlehurst, L., Ly, S., Lu, R., Leong, S., Bengtsson, N., Johansson, B., Riddick, F., Lee, T., Shao, G., McLean, C., and Salour, A., 2008. "Implementation of core manufacturing simulation data in aerospace industry”. In WSC '08: Proceedings of the 40th Conference on Winter Simulation, Winter Simulation Conference.

[16] Lee, Y. T., Leong, S., Riddick, F., Johansson, M., and Johansson, B., 2007. "A pilot implementation of the core manufacturing simulation data information model". In Proceedings of the Systems Interoperability Standards Organization 2007 Fall Simulation Interoperability Workshop.

[17] Skoogh, A., and Johansson, B., 2008. "A methodology for input data management in discrete event simulation projects". In Proceedings of the Winter Simulation Conference, S. J. Mason, R. R. Hill, L. Mnch, O. Rose, T. Jefferson, and J. W. Fowlers, eds., Institute of Electrical and Electronic Engineers, Inc., pp. 2664-2671.

[18] Han, J., and Kamber, M., 2001. Data Mining: Concepts and Techniques. Morgan Kaufman, New York. 
[19] Kantardzic, M., 2011. Data Mining Concepts, Models, Methods, and Algorithms - 2nd Edition. Wiley-IEEE, New Jersey.

[20] Chapman, W. W., 2002. Modern machine shop's handbook for the metalworking industries. Hanser Gardner Publications, Cincinnati, Ohio.

[21] Wang, K., Tong, S., Roucoules, L., and Eynard, B., 2008. "Analysis of data quality and information quality problems in digital manufacturing". Proceedings of the 4th IEEE International Conference on Management of Innovation and Technology, ICMIT, pp. $439-443$.

[22] U.S. Department of Energy, 2004. Energy Use, Loss And Opportunities Analysis: U.S. Manufacturing and Mining. 